\title{
ON THE EXISTENCE OF TRIVIAL INTERSECTION SUBGROUPS
}

BY

\author{
MARK P. HALE, JR.
}

\begin{abstract}
Let $G$ be a transitive nonregular permutation group acting on a set $X$, and let $H$ be the subgroup of $G$ fixing some element of $X$. Suppose each nonidentity element of $H$ fixes exactly $b$ elements of $X$. If $b=1, G$ is a Frobenius group, and it is well known that $H$ has only trivial intersection with its conjugates. If $b>1$, it is shown that this conclusion still holds, provided $H$ satisfies certain natural conditions. Applications to the study of Hall subgroups and certain simple groups related to Zassenhaus groups are given.
\end{abstract}

1. A transitive permutation group $G$ in which every nonidentity element fixes either one or no letters is called a Frobenius group. If $H$ is the stabilizer of a single letter in such a group, it is easy to see that $H$ is a trivial intersection (t.i.) subgroup of $G$, that is $H \cap H^{x}=1$ unless $H=H^{x}$. Theorem A gives a condition for the existence of t.i. subgroups in permutation groups which are generalizations of Frobenius groups.

If $\pi$ is a set of primes, $x$ is a $\pi$-element of a group $G$ if $o(x)$, the order of $x$, is divisible only by primes in $\pi$. A subgroup $H$ of $G$ is a $\pi$-subgroup if every element of $H$ is a $\pi$-element; if also the order $|H|$ of $H$ is prime to the index $|G: H|$ of $H$ in $G$, $H$ is a $\pi$-Hall subgroup. Let $\pi^{\prime}$ denote the collection of all primes not in $\pi$. A group $G$ is called $\pi$-isolated if no $\pi$-element commutes with a $\pi^{\prime}$-element.

Our main theorem is

TheORem A. Let $G$ be transitive on $X$. Suppose that the subgroup $H$ of $G$ of all elements fixing one letter in $X$ is $\pi$-Hall in $G$, and $G$ is $\pi$-isolated. Then if every nonidentity element of $H$ fixes exactly $b$ letters, $H$ is a trivial intersection subgroup of $G$. If $b>1, H$ is also nilpotent, and $\left|N_{G}(H): H\right|=b$.

The proof of this result depends on the description of partitioned groups, given in $\S 2$. In $\S 3$, the proof of Theorem A is given, and we conclude with some applications of this result.

All groups considered are finite. Basic notation, and statements of theorems quoted by name, may be found in [7]. We note that a group $G$ is a Frobenius group if and only if $G$ contains a proper trivial intersection subgroup $H$ which is its own normalizer in $G$. A classical theorem of Frobenius' asserts that in this case, $G$

Presented to the Society, April 18, 1970; received by the editors April 27, 1970.

AMS 1969 subject classifications. Primary 2020; Secondary 2029, 2043.

Key words and phrases. Trivial intersection subgroup, Frobenius group, partitioned groups, Hall subgroup, centralizer of each of its nonidentity elements, exceptional characters.

Copyright $(\mathbb{1}$ 1971, American Mathematical Society 
contains a normal subgroup $K$, with the properties $K \cap H=1, K H=G$. We will call $K$ the Frobenius kernel and $H$ the Frobenius complement of $G$.

The author wishes to express his deep gratitude to Professor Michio Suzuki for many helpful conversations. A preliminary version of parts of this paper was submitted as part of a doctoral dissertation at the University of Illinois, Urbana.

2. A partition of a group $G$ is a collection $\sigma$ of subgroups of $G$ such that every nonidentity element of $G$ occurs in one and only one subgroup in the set $\sigma$. A partition $\sigma$ is called nontrivial if it does not consist of $\{G\}$ alone; $\sigma$ is normal if, for each $U$ in $\sigma, U^{x}$ is in $\sigma$, for all $x$ in $G$. If $\sigma, \tau$ are partitions of $G$ and $g$ is in $G$, then $\sigma^{g}=\left\{U^{g}: U \in \sigma\right\}$ and $\sigma \cap \tau=\{U \cap V: U \in \sigma, V \in \tau\}$ are also partitions of $G$. It is thus evident that any group admitting a nontrivial partition admits a nontrivial normal partition. If $U$ is contained in $\sigma$, a partition of $G$, we call $U$ a component of $\sigma$. The components of a normal partition are t.i. subgroups.

Combining results due to Baer, Kegel, and Suzuki, we may give the following description of groups admitting nontrivial normal partitions.

THEOREM 1. Let $G$ be a finite group having a nontrivial normal partition. Then one of the following cases holds:

(a) $G$ is isomorphic to a Suzuki group $\operatorname{Sz}(q)$ or a linear fractional group $\operatorname{PSL}(2, q)$.

(b) $G$ is isomorphic to $S_{4}$, the symmetric group on four letters.

(c) $G$ is a Frobenius group.

(d) $G$ is a p-group.

(e) $G$ contains a nilpotent normal subgroup $K$ of prime index $p$ in $G$, every element of $G$ not in $K$ has order $p$ and is centralized only by $p$-elements, and $p$ divides $|K|$.

Proof. Let $N$ be the maximal nilpotent normal subgroup of $G$. If $N=1, G$ is nonsolvable; then by Suzuki [13], case (a) holds. If $N$ is nontrivial but contains no Sylow subgroup of $G$, Theorem B and Theorem A of Baer [2] give case (b). Finally, if $N$ contains a Sylow subgroup of $G$, then one of the remaining cases must hold, by Baer [1, Satz 5.1].

We will also need the following lemmas from [1].

LEMMA 1. If $G$ is a Frobenius group, then a Frobenius complement is a component in any nontrivial normal partition of $G$.

LEMMA 2. If $\sigma$ is a partition of $G$, and $x$ and $y$ are commuting elements of $G$ of distinct orders, then $x, y$ are in the same component of $\sigma$.

Proof. We may assume by symmetry that $o(x)<o(y)$; let $m=o(x)$. Then $(x y)^{m}$ $=y^{m} \neq 1$. Since $x y$ and $y$ have a common power $y^{m} \neq 1, x y, y$ and hence $x$ lie in the same component of $\sigma$.

3. The following theorem, due to Suzuki, is needed for the proof of Theorem A.

THEOREM 2. Let $P$ be a $p$-Sylow subgroup of $G$. Suppose that for any $x \neq 1$ in $P$, $C_{G}(x)$ is a p-group, and $x^{p}=1$; then $P$ is a t.i. subgroup of $G$. 
Proof. If $P$ is abelian, $P$ is t.i. since for each $x \neq 1$ in $P, P=C_{G}(x)$. For $p=2, x^{2}=1$ for all $x$ implies $P$ is abelian, so we need only consider $p$ odd, and $P$ nonabelian.

If $P$ contains any nonidentity element conjugate to its inverse, $P$ is abelian, by Theorem (4D) of Brauer and Fowler [4]. Let $D$ be maximal among all nontrivial intersections of $P$ with one of its distinct conjugates $P^{\prime}$, and define $M=N_{G}(D)$. Since $P$ is nonabelian, $M$ has odd order, and is solvable by the Theorem of Feit and Thompson [6]. Since the $p^{\prime}$-elements of $M$ centralize no nonidentity element of $D$, the $q$-Sylow subgroups of $M$ are cyclic for $q \neq p$ [7, p. 200]. Theorem B of Hall and Higman [8] now applies to $M$; thus there is a normal $p^{\prime}$-subgroup $K$ of $M$ such that $M / K$ has a normal $p$-Sylow subgroup. Since $K$ must then centralize $D$, $K=1$, and $M$ has a normal $p$-Sylow subgroup. However, $P$ and $P^{\prime}$ can be chosen so that $P \cap M$ and $P^{\prime} \cap M$ are $p$-Sylow subgroups of $M$. Thus $P \cap M=P^{\prime} \cap M$ $=D$, and $D=N_{P}(D)$, contrary to $D \neq P$. Thus $P$ is a t.i. subgroup of $G$.

Throughout the rest of this section we will assume the following hypothesis:

(H1) $G$ is a transitive permutation group acting on the set $X$. The subgroup $H$ of all elements fixing some element $\alpha$ of $X$ is a $\pi$-Hall subgroup of $G$, and $G$ is $\pi$-isolated. Every element $x \neq 1$ of $H$ fixes exactly $b$ elements of $X, b>1$.

Lemma 3. If (H1) holds for $G, b$ is $a \pi^{\prime}$-number, and every $\pi$-element of $G$ fixes $b$ letters of $X$.

Proof. Let $p$ be a prime in $\pi$, and let $x$ be an element of order $p$ in $H$. The set $X$ contains $|G: H|$ elements, and $x$ fixes $b$ elements and permutes the rest in orbits of length $p$. Thus $p$ divides $|G: H|-b$. Since $p$ is relatively prime to $|G: H|, p$ is relatively prime to $b$.

For the second part, suppose $x$ is a $\pi$-element fixing no points in $X$. Then $x$ is not of prime-power order, for then a conjugate of $x$ would lie in a Sylow subgroup of $H$. Thus there are commuting $\pi$-elements $u, v$ of coprime order with $x=u v$. By induction on $o(x)$, we may assume $u$ and $v$ both fix points of $X$. If $u$ has no fixed points in common with $v, u$ permutes the fixed points of $v$ in orbits of length $o(u)$, and thus $o(u)$ divides $b$, a contradiction. Thus $u$ and $v$ have a common fixed point, which is a fixed point of $x=u v$, proving the lemma.

The next lemma allows us to apply $\S 2$ to our problem.

Lemma 4. Suppose $G$ satisfies (H1). If $H$ is not a t.i. subgroup of $G, G$ contains a collection of t.i. subgroups which induce a nontrivial normal partition of $H$.

Proof. Let $x \neq 1$ be any $\pi$-element of $G$, and let $D_{x}$ denote the subgroup of all elements of $G$ fixing the fixed points of $x$. Let $x$, and $y$ be $\pi$-elements of $G$. Then if $t \neq 1$ is in both $D_{x}$ and $D_{y}, x$ and $y$ have the same $b$ fixed points, so $D_{x}=D_{y}$. Thus the subgroups $D_{x}$ are t.i. subgroups of $G$. If $H \neq D_{x}$ for some $x, H$ is nontrivially partitioned by the subgroups $D_{h}, h \neq 1$ in $H$.

Proof of Theorem A. If $b=1$, the theorem is the classical case; so we assume $b>1$. If $H=1, H$ is a t.i. subgroup; the last paragraph of the proof below applies. 
We adopt ( $\mathrm{H} 1)$ on $G$; suppose $H$ is not a t.i. subgroup of $G$. Then $H$ is partitioned, and one of the cases of Theorem 1 describes $H$. We will show that none of the cases (a)-(e) can occur.

(i) $H$ is not $S z(q), P S L(2, q)$, or $S_{4}$. The possible partitions of these groups are given in Suzuki [13] and Baer [2]. If $H$ is one of these groups, a cyclic Hall subgroup $K$ of odd order is a component of the partition of $H$, and $N_{H}(K)$ is a dihedral group. By a theorem of Jordan [15, p. 6], $N_{G}(K)$ is transitive on the $b$ fixed points of $K$, so $b=\left|N_{G}(K): N_{H}(K)\right|$. Since the automorphism group of an odd-order cyclic group is abelian, and $b \neq 1$, this contradicts the $\pi$-isolation of $G$.

(ii) $H$ is not a Frobenius group. If $H$ is a Frobenius group, a Frobenius complement $C$ is a component of the partition of $H$, by Lemma 1 . Let $K$ be the Frobenius kernel of $H$; by a theorem of Thompson [14], $K$ is nilpotent. The partition of $H$ induces a partition of $K$. We will show that there is a unique component $U$ of the partition containing $Z(K)$. Let $x \neq 1$ be contained in $Z(K)$. If there is an element $y \neq 1$ in $K$ with $o(x) \neq o(y), x$ and $y$ lie in the same component $U$ by Lemma 2 . Then since $U$ contains two elements of unequal order, $Z(K) \leqq U$. Otherwise every element in $K$ has order $p$, and $K$ is a $p$-Sylow subgroup of $H$ and hence of $G$. If any $p^{\prime}-$ element centralized a $p$-element, $K$ would contain $p^{\prime}$-elements. Thus the hypotheses of Theorem 2 hold for $K$, and $K$ is a t.i. subgroup of $G$. Now choose $k \neq 1$ in $K$, and regarding the points of $X$ as right cosets of $H$, let $H t$ be a point fixed by $k$. Then $H t k=H t$, so $t k t^{-1} \in H$. Since $K$ is the unique $p$-Sylow subgroup of $H, t k t^{-1} \in K$, and since $K$ is a t.i. subgroup, $t K t^{-1}=K$. It now is clear that $H t K=H t$, so any fixed point of $k$ is a fixed point for $K$, that is $K=U$ is a component of $H$ containing $Z(K)$.

Now consider the (unique) component $U$ containing $Z(K)$. Since $Z(K)$ is a characteristic subgroup of $K, U$ is normal in $H$. Thus $U$ is normalized by the complement $C$ of $H$. By the previously-cited theorem of Jordan, $M=N_{G}(U)$ is transitive on the fixed points of $U$, so $H<M$, and $M$ contains $\pi^{\prime}$-elements. If $C=N_{M}(C)$, then $M$ is a Frobenius group with complement $C$, since $C$ is a t.i. subgroup of $G$. The Frobenius kernel of $M$ is nilpotent by Thompson's Theorem, so there are $\pi^{\prime}$-elements commuting with $\pi$-elements in $G$, contrary to $\pi$-isolation. However, if there is a $\pi^{\prime}$-element $x$ of $M$ normalizing $C, x$ normalizes $C U$, and since $x$ centralizes no $\pi$-element, $C U$ is nilpotent, contrary to hypothesis. Finally suppose $N_{M}(C)$ contains a $\pi$-element $x$ of prime power order. Then $x$ permutes the $b$ fixed points of $C$, and by Lemma 3, must fix one of them. Thus $\langle x, C\rangle$ is isomorphic to a subgroup of $H$; since $N_{H}(C)=C$, we have $x$ in $C$. Thus $N_{M}(C) \neq C$ is impossible; this disposes with the present case.

(iii) $H$ is a t.i. subgroup. We have shown that if this is false, $H$ is partitioned, and only cases (d) and (e) of Theorem 1 can possibly occur. In both these cases, $Z(H) \neq 1$. Let $x$ be an element of prime order $p$ in $Z(H)$. By $\pi$-isolation, $H=C(x)$, so we may regard $X$ as the set of all conjugates $x^{t}$ of $x, t$ ranging over $G$. The permutation condition in (H1) now becomes 
(H2) Every element $y \neq 1$ of $H$ centralizes $b$ conjugates of $x$.

In particular, $x$ satisfies (H2), so $H$ contains $b$ conjugates of $x$. Let $D$ be the component of the partition of $H$ containing $x$. We will show that $H$ is partitioned by conjugates of $D$. Let $y \neq 1$ be any element of $H$. If $o(y) \neq p$, then $y$ is in $D$, by Lemma 2. Suppose $o(y)=p$. If $C_{G}(y)$ is not a $p$-group, there is a $p^{\prime}$-element $z$ with $y z=z y$. By $\pi$-isolation, $z$ is a $\pi$-element, and hence $y z$ has fixed points, which must be the same as those of $y$ and $z$. Thus $z$ centralizes $x$, and $y, z$, and $x$ all lie in the same component $D$. If $o(y)=p$, and $C_{G}(y)$ is a $p$-group, $C_{G}(y)$ lies in some $p$-Sylow subgroup $P$ of $G$. Since $H$ is $\pi$-Hall, for some $t, C_{G}(y) \leqq P \leqq H^{t}$. By (H2), $X \cap C_{G}(y)$ and $X \cap H^{t}$ both contain $b$ conjugates of $x$. Thus $y$ and $x^{t}$ have the same fixed points, and $y$ lies in $D^{t}$.

Since $H$ is partitioned by isomorphic subgroups, from Theorem 1 and Lemma 3 we see that $H$ is a $p$-group which satisfies $z^{p}=1$ for all $z$ in $H$. Now $H$ satisfies the hypothesis of Theorem 2, so $H$ is a t.i. subgroup of $G$.

We have proven that $H$ is a t.i. subgroup. It then follows that all elements of $H$ fix the same $b$ letters, so by the theorem of Jordan, $b=\left|N_{G}(H): H\right|$. As $b \neq 1$, and $H$ is a $\pi$-Hall subgroup, $H$ is normalized by $\pi^{\prime}$-elements, which centralize no elements of $H$. Thus $N_{G}(H)$ is a Frobenius group, and $H$ is nilpotent by Thompson's Theorem. This completes the proof of Theorem A.

The hypotheses of Theorem A cannot be weakened significantly without invalidating the conclusion. For the following examples, let $G$ be the group $\operatorname{PSL}(2,11)$, the structure of which can be found in [10, II.8]. Consider the transitive representation of $G$ on right cosets $\{H x\}$ of a 2-Sylow subgroup $H$. Each element of $H$ fixes 9 cosets, but $H$ is not a t.i. subgroup, since $G$ contains a subgroup $D$ isomorphic to a dihedral group of order 12 .

For $\pi=\{2,3\}, D$ is a $\pi$-Hall subgroup and $G$ is $\pi$-isolated, but $D$ is not a t.i. subgroup. Even the requirement that $G$ is $\pi$-isolated, contains a $\pi$-Hall subgroup, and contains subgroups partitioning the $\pi$-elements is not enough. The subgroups $B$ isomorphic to $\operatorname{PSL}(2,5)$ contained in $\operatorname{PSL}(2,11)$ illustrate this. In spite of these examples, the use of $\S 2$ in studying permutation groups in which any element $x \neq 1$ fixing one letter fixes $b$ letters might lead to a partial description of these groups.

4. As an application of our previous results, we give a criterion for the existence of abelian Hall subgroups.

THEOREM B. If $G$ is a group in which the centralizer of every nonidentity $\pi$ element is a $\pi$-Hall subgroup, $G$ has an abelian $\pi$-Hall subgroup which is the centralizer of each of its nonidentity elements.

Proof. It is enough to show $G$ has an abelian $\pi$-Hall subgroup, as the remainder of the conclusion follows easily. Suppose first that $\pi$ contains more than one prime. Let $x$ be a $\pi$-element of prime order $p$, and let $Q$ be any $q$-Sylow subgroup of $C(x), q \neq p$. If $y \neq 1$ is any element in $Q, C(x y)=C(x)=C(y)$, since $x$ and $y$ are powers of $x y$. Thus $Q \leqq Z(C(x))$, and the conclusion follows, by interchanging $p$ 
and $q$. If $\pi=\{p\}$, every $p$-element of $G$ is central in some $p$-Sylow subgroup of $G$. If $b$ is the number of conjugates of some $p$-element $x \neq 1$ occurring in any $p$-Sylow subgroup $P$, then every nonidentity $p$-element centralizes $b$ conjugates of $x$. Thus Theorem A applies, and $P$ is a t.i. subgroup of $G$. Now $P$ centralizes each of its nonidentity elements, and the conclusion follows.

Theorem A also has applications in the study of certain simple groups. We call a subgroup $A$ of a group $G$ a special subgroup if $A$ is the centralizer of each of its nonidentity elements, and $\left|N_{G}(A): A\right|=2$.

Groups having special subgroups have been studied in [5], [9], and [11]; the known simple groups with this property are the groups $\operatorname{Sz}(q), P S L(3,4)$, and $\operatorname{PSL}(2, q)$. Feit and Thompson determined those simple groups having a special subgroup of order 3 . We will describe our results here, referring the reader to [9] for the necessary preliminaries.

Let $G$ be a simple group containing a special subgroup of order $a \geqq 5$. Applying the method of exceptional characters to $G$, we obtain $(a-1) / 2$ "exceptional" irreducible characters, and one "distinguished" irreducible character $\theta$ of $G$.

Employing knowledge of these characters and an idea of Suzuki's [12], a formula for $|G|$ may be given. This formula is $|G|=a \theta(1)(\theta(1)+\varepsilon) r^{2}$, where $\varepsilon=1$ or -1 , and $r$ is an integer with $r^{2} \equiv 1$ modulo $a$. If $r=1$ (this is the case in the known simple groups listed above), then much more information can be obtained on the conjugacy classes of $G$. In particular, every element has order dividing $a, \theta(1)$ or $\theta(1)+\varepsilon$. In this context, we offer the following generalizations of Theorem 1 of [9].

THEOREM C. Let $G$ be a simple group having a special subgroup of order $a \geqq 5$. If in the group order formula $|G|=a \theta(1)(\theta(1)+\varepsilon) r^{2}, r=\varepsilon=1$, and $G$ has a subgroup of order $\theta(1)$, then $G$ is isomorphic to either $\operatorname{Sz}(q)$ or $\operatorname{PSL}(2, q)$, with $q=\theta(1)$.

REMARK. If $\theta(1)$ is even, or $\theta(1)$ is odd and $\theta(1)-\varepsilon \leqq 22 a$, it is easy to show that $G$ contains an element $x$ with $\left|C_{G}(x)\right|=\theta(1)$, using the information on conjugacy classes given in [9].

Description of proof. Let $D$ be the subgroup of order $\theta(1)$ of $G$. Employing the known values of the exceptional characters of $G$, it can be seen that $G$ satisfies the hypotheses of Theorem A with respect to the permutation representation of $G$ on cosets of $D$, with $b=|A|$. It is then easy to show that the permutation character of $G$ acting on right cosets of $N_{G}(D)$ is $1+\theta$, so that $G$ is doubly transitive. As the values of $\theta$ are known, we can show that in this representation, $G$ is a Zassenhaus group. The theorem then follows from the classification of these groups.

THEOREM D. If $G$ is a simple group with a special subgroup of order a, and for some involution $x$ in $G,\left|C_{G}(x)\right| \leqq 9 a / 2$, then $G$ is isomorphic to $\operatorname{PSL}(2, q)$, for some $q$.

Description of proof. Using an idea of Brauer's [3], we can show that $\left|C_{G}(x)\right|$ $\leqq 9 a / 2$ forces $r=1$ in the group order formula, aside from cases involving small values of $a$, which can be eliminated by separate arguments. Then by considering 
the distribution of conjugacy classes, we can see that every element of order dividing $\theta(1)$ has a centralizer of order $\theta(1)$, and that Theorem B applies to $G$. By considering elementary divisibility properties, we see that $|G|=a(2 a+\varepsilon)(2 a+2 \varepsilon)$, and the conclusion follows from Theorem 1 of [9].

\section{BIBLIOGRAPHY}

1. R. Baer, Partitionen endlicher Gruppen, Math. Z. 75 (1960/61), 333-372. MR 27 \#1492.

2. - Einfache Partitionen endlicher Gruppen mit nichttrivialer Fittingscher Untergruppe, Arch. Math. 12 (1961), 81-89. MR 25 \#115.

3. R. Brauer, Some applications of the theory of blocks of characters of finite groups. III, J. Algebra 3 (1966), 225-255. MR 34 \#2716.

4. R. Brauer and K. Fowler, On groups of even order, Ann. of Math. (2) 62 (1955), 565-583. MR 17, 580.

5. W. Feit and J. G. Thompson, Finite groups which contain a self-centralizing subgroup of order 3, Nagoya Math. J. 21 (1962), 185-197. MR 26 \#192.

6. - Solvability of groups of odd order, Pacific J. Math. 13 (1963), 775-1029. MR 29 \#3538.

7. D. Gorenstein, Finite groups, Harper \& Row, New York, 1968. MR 38 \#229.

8. P. Hall, and G. Higman, On the p-length of p-soluble groups and reduction theorems for Burnside's problem, Proc. London Math. Soc. (3) 6 (1956), 1-42. MR 17, 344.

9. K. Harada, A characterization of the groups LF (2, q), Illinois J. Math. 11 (1967), 647-659. MR 36 \#1529.

10. B. Huppert, Endliche Gruppen. I, Die Grundlehren der math. Wissenschaften, Band 134, Springer-Verlag, Berlin and New York, 1967. MR 37 \#302.

11. W. B. Stewart, Strongly self centralizing 3-centralizers (to appear).

12. M. Suzuki, Applications of group characters, Proc. Sympos. Pure Math., vol. 6, Amer. Math. Soc., Providence, R. I., 1962, pp. 101-105. MR 24 \#A3196.

13. - On a finite group with a partition, Arch. Math. 12 (1961), 241-254. MR 25 \#113.

14. J. G. Thompson, Normal p-complements for finite groups, J. Algebra 1 (1964), 43-46. MR 29 \#4793.

15. H. Wielandt, Finite permutation groups, Lectures, University of Tübingen, 1954/55; English transl., Academic Press, New York, 1964. MR 32 \#1252.

SOUTHERN ILLINOIS UNIVERSITY, CARbONDALe, IllinoIs 62901 